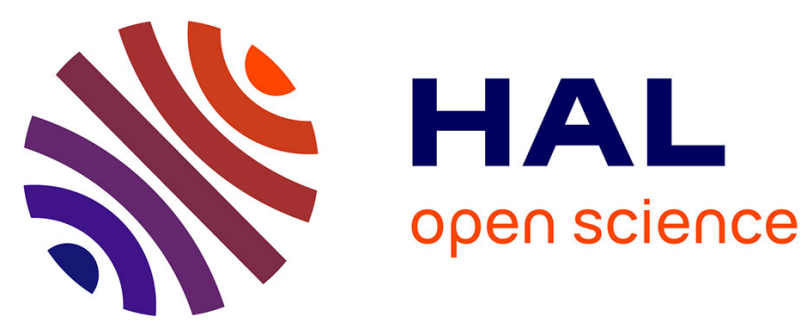

\title{
Robustness of Cellular Automata in the Light of Asynchronous Information Transmission
}

\author{
Olivier Bouré, Nazim A. Fatès, Vincent Chevrier
}

\section{To cite this version:}

Olivier Bouré, Nazim A. Fatès, Vincent Chevrier. Robustness of Cellular Automata in the Light of Asynchronous Information Transmission. 10th International Conference on Unconventional Computing, Jun 2011, Turku, Finland. pp.52-63, 10.1007/978-3-642-21341-0_11 . inria-00563904v2

\section{HAL Id: inria-00563904 https://hal.inria.fr/inria-00563904v2}

Submitted on 30 Mar 2011

HAL is a multi-disciplinary open access archive for the deposit and dissemination of scientific research documents, whether they are published or not. The documents may come from teaching and research institutions in France or abroad, or from public or private research centers.
L'archive ouverte pluridisciplinaire HAL, est destinée au dépôt et à la diffusion de documents scientifiques de niveau recherche, publiés ou non, émanant des établissements d'enseignement et de recherche français ou étrangers, des laboratoires publics ou privés. 


\title{
Robustness of Cellular Automata in the Light of Asynchronous Information Transmission
}

\author{
Olivier Bouré, Nazim Fatès, Vincent Chevrier \\ \{olivier.boure, nazim.fates, vincent.chevrier\}@loria.fr \\ Nancy Université - INRIA Nancy-Grand-Est - LORIA
}

March 30, 2011

\begin{abstract}
Cellular automata are classically synchronous: all cells are simultaneously updated. However, it has been proved that perturbations in the updating scheme may induce qualitative changes of behaviours. This paper presents a new type of asynchronism, the $\beta$-synchronism, where cells still update at each time step but where the transmission of information between cells is disrupted randomly. We experimentally study the behaviour of $\beta$-synchronous models. We observe that, although many effects are similar to the perturbation of the update, novel phenomena occur. We particularly study phase transitions as an illustration of a qualitative variation of behaviour triggered by continuous change of the disruption probability $\beta$.
\end{abstract}

Keywords: asynchronous cellular automata, discrete dynamical systems, robustness, phase transitions, directed percolation

\section{Introduction}

Cellular automata are a parallel, spatially-extended, model of computation, which has been studied as an alternative to the sequential computing models, for instance Turing machines. By their very structure, they are well-suited for modelling natural phenomena and for the design of massively parallel algorithms. These mathematical objects have been mostly considered in the synchronous case, that is, when all their components are simultaneously updated. However, this hypothesis of perfect synchrony is somehow inadequate when modelling systems that are subject to noise or non-ideal information transmission, as often met in various natural systems or in asynchronous parallel computing devices. This is why authors tackled the question of whether a cellular automaton is robust to non-ideal updating, either without changing its local transition rule (e.g. [4]), or by adding adapted constructs (e.g. [8]). 
The aim of this paper is to study the robustness of cellular automata by considering the possibility of disruptions in the transmission of information between cells. To this end, we describe the updating process of the system in the frame of a cellular cycle. This cycle consists of two steps: (a) the local computation and (b) the transmission of the updated state of a cell to its neighbours. This dichotomy induces two related types of asynchronism: the update-wise asynchronism, or $\alpha$-synchronism, which disrupts step (a) and a novel type of asynchronism, the influence-wise asynchronism or $\beta$-synchronism, which disrupts step (b) and perturbs the interaction between a cell and its neighbours.

The $\alpha$-synchronous updating is now a relatively well-studied perturbation, whose effect can either be "inoffensive" or trigger drastic qualitative changes, such as phase transitions $[2,3,6]$. Our goal is now to examine the effects of $\beta$-synchronism on simple cellular automata, in particular, in comparison with those of $\alpha$-synchronism. We aim at extending the range of perturbations, in order to gain insight on how complex collective behaviour emerges from numerous simple local interactions.

On the formal side, this type of asynchronism leads us to extend the cell state space in order to distinguish the eigenstate, the actual state of the cell, and the observable state, the state of the cell which is observed by its neighbours. The corresponding definitions are introduced in Sec. 2 while experimental observations are presented in Sec. 3. We then analyze more particularly the occurrence of phase transitions in Sec. 4 and proceed to bring discussion in Sec. 5 .

\section{Asynchronous Cellular Automata}

\subsection{Cellular Automata}

A synchronous cellular automaton is a discrete dynamical system defined by $A=\{\mathcal{L}, Q, \mathcal{N}, f\}$ where :

- $\mathcal{L} \subset \mathbb{Z}^{d}$ the array of the cellular space, where an element of $\mathcal{L}$ represents a cell.

- $Q$ is a nonempty finite set of states.

- $\mathcal{N} \subset \mathcal{L}$ is a finite set of vectors called the neighbourhood, which associates to a cell the set of its neighbouring cells. $\mathcal{N}$ and $\mathcal{L}$ are such that for all $c \in \mathcal{L}$ and for all $n \in \mathcal{N}$, the neighbour $c+n$ is in $\mathcal{L}$.

- $f$ is the local transition rule, which defines the next state of a cell according to the state of this cell and the ones of its neighbours.

A configuration $x^{t}$ represents the state of the automaton at time $t$; it is defined as a function $x^{t}: \mathcal{L} \rightarrow Q$ which maps each cell to a state. Classically, cellular automata are synchronously updated, meaning that at each time the local transition rule is applied simultaneously of all cells. The global transition function is therefore defined as $x^{t+1}=F\left(x^{t}\right)$, so that, for $\mathcal{N}=\left\{n_{1}, \ldots, n_{k}\right\}$ :

$$
\forall c \in \mathcal{L}, x^{t+1}(c)=f\left(x^{t}(c), x^{t}\left(c+n_{1}\right), \ldots, x^{t}\left(c+n_{k}\right)\right) .
$$




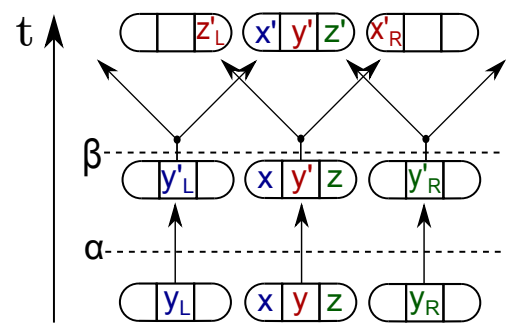

Figure 1: Representation of a cell cycle, for a cell with two neighbours, denoted by indices L and R. A triplet represents a cell with its state $(y)$ and the observable states of its neighbours ( $x$ for left cell and $z$ for right). The prime sign indicates states that are updated. Solid arrows show the updates of states, otherwise states are conserved from the previous step. In $\alpha$-synchronism, the local transition update applies with probability $\alpha$ and in $\beta$-synchronism, the information transmission is applied with probability $\beta$. The lower arrow is the local transition update, which performs $y^{\prime}=f(x, y, z)$ when applied. The forking upper arrow is the information transmission, which performs $z_{\mathrm{L}}^{\prime}=y^{\prime}$ and $x_{\mathrm{R}}^{\prime}=y^{\prime}$ when applied.

Without loss of generality, we assume that the neighbourhood $\mathcal{N}$ does not contain the cell itself. This hypothesis is necessary to explicitly represent the flow of information between a cell and its neighbours. Note that this does not restrict the expressiveness of $f$ since the current state of a cell $x^{t}(c)$ is always a parameter of $f$, possibly not taken into account in the transition calculus.

\subsection{Asynchronism as a disruption of cell activity}

Cell cycle. The update of a cell can be represented by a cell cycle, which we decompose into two steps (see Fig. 1):

- the state update step, where a cell changes its state according to the local transition function.

- the information transmission step, where the cell transmits the updated state to its neighbours.

We give an example of the cell cycle for the different updating schemes on Fig. 2. The update-wise asynchronous updating in cellular automata, or $\alpha$ synchronism [3], is defined as follows: at each time step, each cell is updated with a fixed probability $\alpha$, or else left unchanged. We introduce a new type of asynchronism, the $\beta$-synchronism, where each cell is always updated but the transmission of the new state to the neighbourhood is realized with a fixed probability $\beta$. As a result, both perturbations consist in applying one of the two steps of the cell cycle with a probability defined as the synchrony rate. Please note that for the sake of simplicity, $\alpha$ and $\beta$ denote both the type of asynchronism and the associated synchrony rates. 


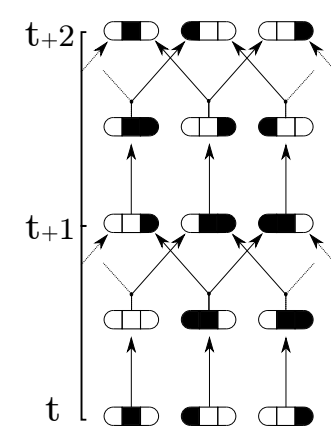

Synchronous

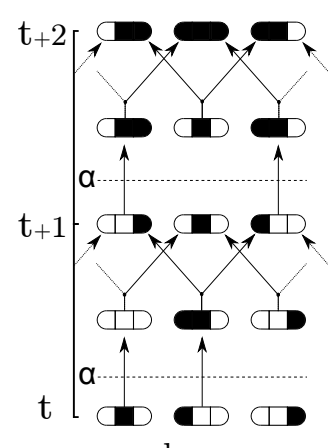

$\alpha$-synchronous

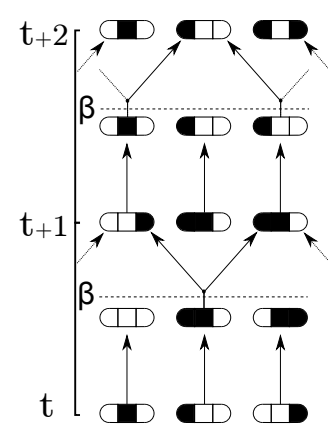

$\beta$-synchronous

Figure 2: Example of the time cycle for a 3-cell sample for synchronous (left), $\alpha$ synchronous (middle) and $\beta$-synchronous (right) updating schemes ( 0 is denoted by a white space, 1 by black). The rule is ECA 50 (see Sec. 2.3) but is not important here.

Formally, we introduce a selection function $\Delta_{\alpha}: \mathbb{N} \rightarrow \mathcal{P}(\mathcal{L})$ which returns for time $t$ the subset of cells to be updated, where each cell has a probability $\alpha$ to be selected. Note that when $\alpha=1$ the updating is fully synchronous and the system is deterministic. The global transition function becomes $\forall t \in \mathbb{N}, \forall c \in$ $\mathcal{L}, \mathcal{N}=\left\{n_{1}, \ldots, n_{k}\right\}$ :

$$
f_{\Delta_{\alpha}}\left(x^{t}(c)\right)= \begin{cases}f\left(x^{t}(c), x^{t}\left(c+n_{1}\right), \ldots, x^{t}\left(c+n_{k}\right)\right) & \text { if } c \in \Delta_{\alpha}(t) \\ x^{t}(c) & \text { otherwise. }\end{cases}
$$

$\beta$-synchronism. To define this new asynchronism, we need to extend the classical definition of cellular automata by taking into account the difference between the eigenstate of a cell, and the observable state.

Let us consider a cellular automaton $A=\{\mathcal{L}, Q, \mathcal{N}, f\}$. From $A$ we derive a new cellular automaton $A^{\prime}=\left\{\mathcal{L}, Q^{\prime}, \mathcal{N}, f^{\prime}\right\}$ where :

- $Q^{\prime}=Q^{2}$ is the new set of states.

For a given configuration $x^{t}$, a cell state is denoted by $x^{t}=\left(\begin{array}{l}x_{\mathrm{e}}^{t} \\ x_{\mathrm{o}}^{t}\end{array}\right)$ with $x_{\mathrm{e}}^{t}: \mathcal{L} \rightarrow Q$ the eigenstate of the cell, and $x_{\mathrm{o}}^{t}: \mathcal{L} \rightarrow Q$ the state of the cell observable by its neighbourhood.

- the local transition function $f^{\prime}$ is splitted into two parts to decompose its action: state update and information transmission.

We thus write $f^{\prime}=f_{\mathrm{t}} \circ f_{\mathrm{u}}$, so that:

- $f_{\mathrm{u}}: Q^{\prime k+1} \rightarrow Q^{\prime}$ is the update function, which computes the new state of the cell based on its eigenstate and the observable state of the neighbours:

$$
f_{\mathrm{u}}\left(\left(\begin{array}{c}
e \\
o
\end{array}\right),\left(\begin{array}{c}
\cdot \\
o_{1}
\end{array}\right), \ldots,\left(\begin{array}{c}
\cdot \\
o_{k}
\end{array}\right)\right)=\left(\begin{array}{c}
f\left(e, o_{1}, \ldots, o_{k}\right) \\
o
\end{array}\right)
$$


- $f_{\mathrm{t}}: Q^{\prime} \rightarrow Q^{\prime}$ is the transmission function, which replaces the observable state by the eigenstate:

$$
f_{\mathrm{t}}\left(\left(\begin{array}{l}
e \\
o
\end{array}\right)\right)=\left(\begin{array}{l}
e \\
e
\end{array}\right)
$$

We use a similar selection function $\Delta_{\beta}$ as introduced for $\alpha$-synchronism, where cells are selected with probability $\beta$, and define the global transition function as, $\forall t \in \mathbb{N}, \forall c \in \mathcal{L}, \mathcal{N}=\left\{n_{1}, \ldots, n_{k}\right\}$ :

$$
f_{\Delta_{\beta}}\left(x^{t}(c)\right)= \begin{cases}f_{\mathrm{t}} \circ f_{\mathrm{u}}\left(x^{t}(c), x^{t}\left(c+n_{1}\right), \ldots, x^{t}\left(c+n_{k}\right)\right) & \text { if } c \in \Delta_{\beta}(t) \\ f_{\mathrm{u}}\left(x^{t}(c), x^{t}\left(c+n_{1}\right), \ldots, x^{t}\left(c+n_{k}\right)\right) & \text { otherwise. }\end{cases}
$$

\subsection{Models studied}

Now that we defined our two updating schemes for cellular automata, we choose to study their effects on well-studied models.

The Game of Life. This 2-dimensional cellular automaton is expressed in our formalism as $A_{G L}=\{\mathcal{L}, \mathcal{N},\{0,1\}, f\}$ where:

- $\mathcal{L}=\{\mathbb{Z} / L \mathbb{Z}\} \times\{\mathbb{Z} / L \mathbb{Z}\}$ is a square grid of size $L$ with periodic boundary conditions.

- $\mathcal{N}=\{c \in \mathcal{L},\|c\|=1\}$ represents the 8-cell (Moore) neighbourhood.

- the local transition function $f: Q^{9} \rightarrow Q$ is outer-totalistic, that is, it can be written $f\left(x^{t}(c), x^{t}\left(c+n_{1}\right), \ldots, x^{t}\left(c+n_{8}\right)\right)=\delta\left(x^{t}(c), s\right)$ where $s=$ $\sum_{c^{\prime} \in \mathcal{N}} x^{t}\left(c^{\prime}\right)$.

$$
\begin{aligned}
& \text { If } x^{t}(c)=0, \text { then } \delta\left(x^{t}(c), s\right)=\left\{\begin{array}{ll}
1 & \text { if } s=3 \\
0 & \text { otherwise. }
\end{array} \quad\right. \text { (Birth rule) } \\
& \text { If } x^{t}(c)=1, \text { then } \delta\left(x^{t}(c), s\right)=\left\{\begin{array}{ll}
1 & \text { if } s \in\{2,3\} \\
0 & \text { otherwise. }
\end{array} \quad\right. \text { (Survival rule) }
\end{aligned}
$$

Elementary Cellular Automata (ECA). An ECA is a 1-D binary cellular automaton with nearest-cell neighbourhood, whose transition function is determined according to Wolfram's notation. In our formalism, ECAs are denoted by $A_{E}=\{\mathcal{L}, \mathcal{N},\{0,1\}, f\}$ where:

- $\mathcal{L}=\{\mathbb{Z} / L \mathbb{Z}\}$ is a 1-dimensional ring.

- $\mathcal{N}=\{-1,+1\}$, i.e. the 2-cell neighbourhood.

- the local transition function $\delta$ is determined by its code: $W=f(0,0,0) \cdot 2^{0}+f(0,0,1) \cdot 2^{1}+\ldots+f(1,1,1) \cdot 2^{7}$. 


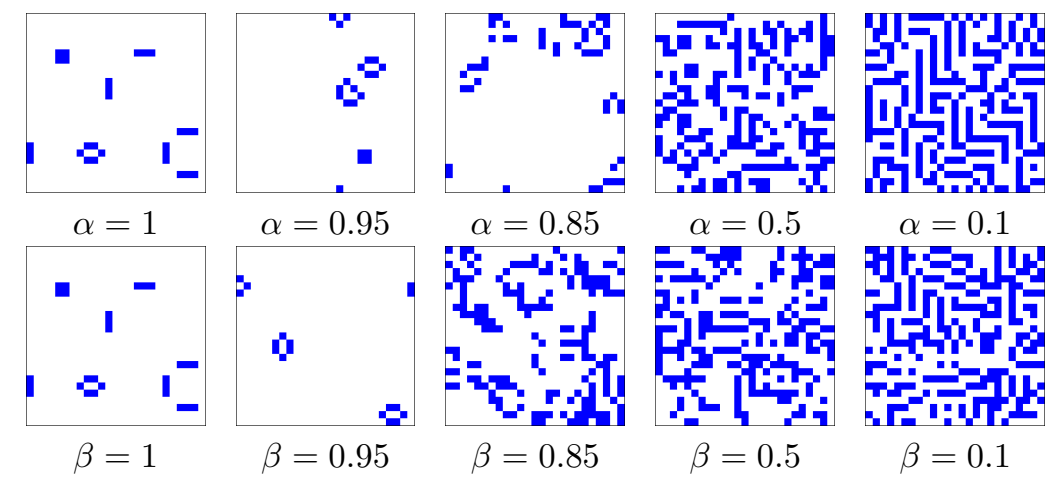

Figure 3: Game of Life configurations for different values of $\alpha$ and $\beta$ (0 is denoted by a white space, 1 by blue). These are obtained with the software FiatLux for identical initial states, for a transient time $t \approx 1000$. The first row shows the different behaviors obtained with different values of $\alpha$-synchronism. The second row displays them for the same values of $\beta$-synchronism.

\section{Qualitative observations}

We now observe qualitatively the effect of $\beta$-synchronism. We are in particular interested in knowing whether its effects will differ from those of $\alpha$-synchronism. In the rest of this paper, initial conditions are constructed by assigning to each cell an equal value to the observable state and the eigenstate, and choosing this value randomly and uniformly in $\{0,1\}$.

\subsection{The Game of Life}

Figure 3 shows sample configurations appearing for random initial conditions after a transient time of 1000 , which was observed sufficient for reaching a steady state. We observe that the behaviour separates into two distinct phases for both types of asynchronism: the system converges to a fixed point for values of the synchrony rate $\alpha$ or $\beta$ higher than 0.9 , whereas a labyrinth-like pattern appears for values lower than 0.9. To quantify this observation, we use the following macroscopic parameters:

- the density is the ratio of cells with state (or eigenstate) 1.

- the activity is the ratio of unstable cells. A cell is said unstable at time $t$ if a synchronous update modifies its state (or eigenstate).

Note that the latter definition cannot be readily transposed for the $\beta$-synchronism. Indeed what does a synchronous update mean for a cell whose eigenstate and observable state are different? As neighbourhood knowledge is always absolute in synchronous cellular automata, we reckon that it should also be the cases for the estimation of activity. Therefore, using the notations defined in Fig. 1, we define a cell as instable if $y \neq f\left(y_{\mathrm{L}}, y, y_{\mathrm{R}}\right)$. 


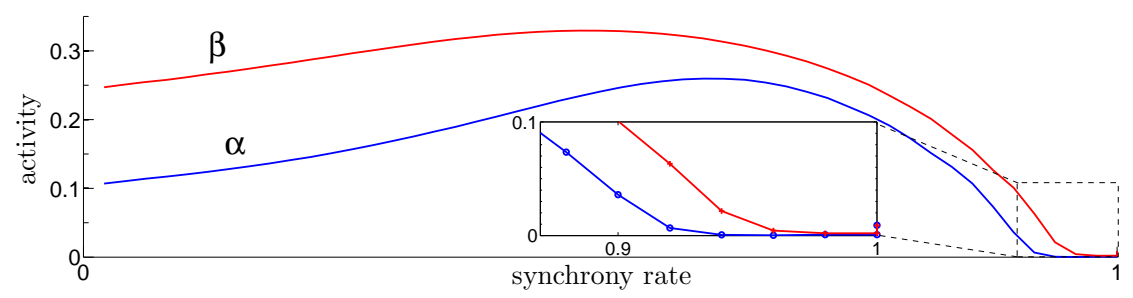

Figure 4: Steady-state values of the activity parameter for the Game of Life with synchrony rates $\alpha$ and $\beta$, averaged over a sample of 50 initial conditions of size $\mathrm{L}=100, t \in[10000,11000]$. Inset: a closeup for values $\alpha, \beta \in] 0.9,1]$.

Figure 4 compares the two types of asynchronism through the activity parameter after a steady state has been reached. At first sight, the $\beta$-synchronous Game of Life seems to react in a similar way to its $\alpha$-synchronous counterpart :

1. A singularity occurs for the activity parameter in the synchronous case $(\alpha, \beta=1)$. This phenomenon has been explained for $\alpha$-synchronism as the loss of stable periodic patterns when a noise is introduced no matter how small $(\alpha<1)$ [1]. The same explanation stands for $\beta$-synchronism. This means that the traditional construct to make the Game of Life Turinguniversal, that is, by using periodical patterns such as gliders, no longer holds. Nevertheless, the question of the Turing-universality of the asynchronous Game of Life still stands.

2. The macroscopic behaviour confirms the phase separation for a critical value of the synchrony rate $\alpha_{c}$ (resp. $\beta_{c}$ ). For values $\alpha>\alpha_{c}$ (resp. $\beta>\beta_{c}$ ), the system converges to a stable fixed point of low density, which constitutes a passive phase. However, for values $\alpha<\alpha_{c}$ (resp. $\beta<\beta_{c}$ ), the system enters an active phase, characterized by the labyrinth pattern (though less regular in $\beta$ - than in $\alpha$-synchronism).

The comparison of both types of asynchronism reveals a similar reaction of the Game of Life, but this calls for a closer observation of the characteristics of the phase transition (see Sec. 4.1).

\subsection{Elementary Cellular Automata}

Through the search of reflexive and complementary symmetries, it is possible to narrow down the number of ECA to study from the 256 possible models to 88 inequivalent ECA. To quantify these observations on the $88 \mathrm{ECA}$, we use the following macroscopic parameters:

- the density $d$, as defined above.

- the 01 block density (00, respectively) is the ratio of successive cells with states $0-1$ (resp., $0-0)$. 


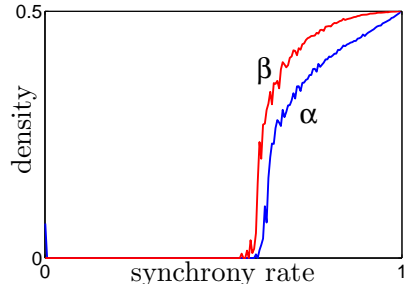

(a) ECA 50, density

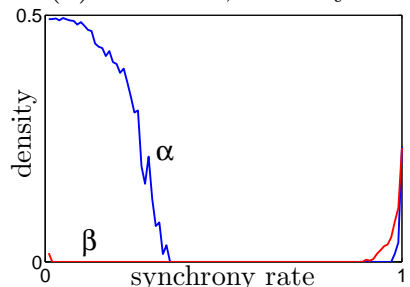

(c) ECA 6, density

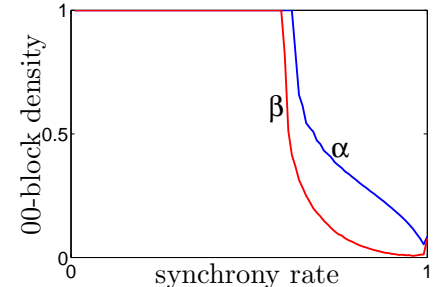

(b) ECA 50, 00-block-density

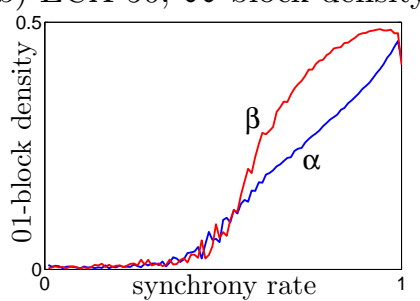

(d) ECA 178, 01-block-density

Figure 5: Steady-state values of various observation parameters for different ECA. The grid size is $F=1000$, transient time is $T_{\mathrm{t}}=\max (10000,1000 / \alpha)$ ( $\beta$ respectively) and averages are obtained for a sampling time $T_{\mathrm{s}}=$ $\max (1000,100 / \alpha)(\operatorname{resp} . \beta)$.

When comparing visually the plot profiles of the 88 minimal ECA under $\alpha$ - and $\beta$-synchronism ${ }^{1}$, it can be observed that most rules display similar reactions to both types of asynchronism. However, some surprising divergences of plot profile appeared for lower synchrony rates, for example for ECA 6, 22, 72 and 200. For the sake of conciseness, we choose to focus on a single rule, namely ECA 50 and leave for future work the exhaustive study of the entire range of phenomena.

The case of ECA 50. A notable phenomenon appears for the $\beta$-synchronous ECA 50. The study of the density parameter (see Fig. 5a) shows a similar plot profile for both $\alpha$ - and $\beta$-synchronism, including a phase transition, which suggests that ECA 50 reacts in a similar way to these two perturbations.

However, a difference of behaviour is observed with the visual inspection of the evolution of the automaton over a few time steps (see Fig. 6). For instance, for the same synchrony rate 0.75 , the patterns in $\beta$-synchronism appear much more regular than $\alpha$-synchronism. In particular, in the long run clusters of 0-states appear less frequently, and 111 patterns seem inexistent.

This can be explained for values of $\beta$ close to 1 with the following observations:

- the checkerboard regions (i.e. alternated 0 s and $1 \mathrm{~s}$ ) are robust to the $\beta$ synchronism, that is, the few anomalies (regions of os) that disturb the

\footnotetext{
${ }^{1}$ for the complete results: http://www.loria.fr/ boure/results/ecaparambeta/
} 


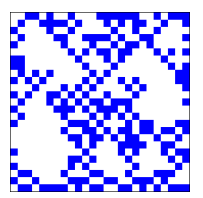

$\alpha=0.75$

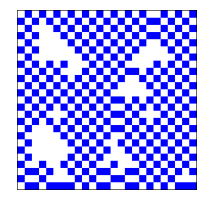

$\beta=0.75$

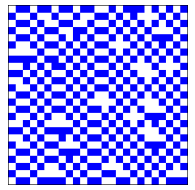

$\alpha=0.95$

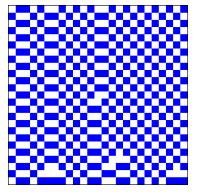

$\beta=0.95$

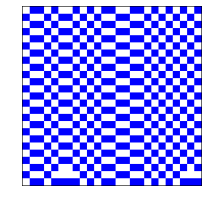

synchronous

Figure 6: Configurations for ECA 50 under different updating schemes ( 0 is denoted by a white space, 1 by blue).

Table 1: Table of probabilities for updated states to become 1 after an $\beta$ synchronous transition of ECA 51 (inversion rule). Each column represents a possible state for a cell and its neighbourhood (upper box) and the associated probability to become a 1 for the output of the transition function (lower box).

Synchronized states

\begin{tabular}{|c|c|c|c|c|c|c|c|}
\hline 0 & 0 & 1 & 1 & . 0 & . 0 & . 1 & . 1 \\
\hline $\begin{array}{lll}0 & 0 & 0\end{array}$ & $\begin{array}{lll}0 & 0 & 1\end{array}$ & $\begin{array}{lll}0 & 1 & 0\end{array}$ & $\begin{array}{lll}0 & 1 & 1\end{array}$ & 100 & $\begin{array}{lll}1 & 0 & 1\end{array}$ & 110 & 111 \\
\hline 1 & 1 & 0 & 0 & 1 & 1 & 0 & 0 \\
\hline$\beta$ & $\beta$ & $1-\beta$ & $1-\beta$ & $\beta$ & $\beta$ & $1-\beta$ & $1-\beta$ \\
\hline
\end{tabular}

Desynchronized states

\begin{tabular}{|c|c|c|c|c|c|c|c|}
\hline . 0 . & . 0 . & . 1 . & . 1 & .0 . & . 0 . & 1. & 1 \\
\hline $\begin{array}{lll}0 & 1 & 0\end{array}$ & $\begin{array}{lll}0 & 1 & 1\end{array}$ & $\begin{array}{lll}0 & 0 & 0\end{array}$ & $\begin{array}{llll}0 & 0 & 1\end{array}$ & $\begin{array}{lll}1 & 1 & 1\end{array}$ & 110 & $\begin{array}{lll}1 & 0 & 0\end{array}$ & $\begin{array}{lll}1 & 0 & 1\end{array}$ \\
\hline 1 & 1 & 0 & 0 & 1 & 1 & 0 & 0 \\
\hline 1 & 1 & 0 & 0 & 1 & 1 & 0 & 0 \\
\hline
\end{tabular}

regularity of the pattern are quickly restored to the original pattern.

- the pairs (i.e. 00 and 11 pairs) follow a non-biased random walk, and annihilate when they meet.

As a consequence, if $L$ is even, in the long run, the system tends to be covered by checkerboard patterns, which is a new property exclusive to $\beta$-synchronism.

This example illustrates the qualitative differences that may occur between $\alpha$ - and $\beta$-synchronous cellular automata. More specifically, we note that ECA 50 is more regular for $\beta$-synchronism than for $\alpha$-synchronism. This regularity can be "seen" as the proximity between rule ECA 50 and the inversion rule ECA 51, which differ only by one bit in their transition table. As the rule ECA 51 is insensitive to $\beta$-synchronism (the inversion rule is independent from the neighbours state), there also seems to exist a proximity in terms of global behaviour between ECA 50 and 51 for the $\beta$-synchronism. One may thus wonder what is the origin of such a radical difference between the two updating schemes. In the next paragraph, we endeavour to capture this difference in the framework of stochastic cellular automata.

Stochastic cellular automata. It is straightforward to describe $\alpha$-synchronous ECA in terms of elementary stochastic cellular automata, that is, to define them 
with a function $f_{\alpha}:\{0,1\}^{3} \rightarrow[0,1]$ which associates to each neighbourhood state $(x, y, z)$ the probability to update to 1 . Indeed, the local rule $f_{\alpha}$ is simply obtained as the barycentre of the transition function $f$ and the identity rule Id with weights $\alpha$ and $1-\alpha$, respectively.

By contrast, $\beta$-synchronism requires us to extend the state space in order to differentiate the eigenstate and the observable state. As a result, the number of inputs of the transition table is doubled to take into account whether the two states are synchronized, that is, if the eigenstate and the observable state are identical.

As said before, ECA 51 is insensitive to $\beta$-synchronism. However, this particular property of ECA 51 does not appear readily on its transition table (see Table 1). This makes the stochastic table all the more "cryptic", as the divergence from synchronism cannot be deduced from the reading of the table, unlike $\alpha$-synchronism. This shows that the difference between the two types of asynchronism is non-trivial, and justifies the experimental approach adopted to study these systems.

\section{Study of phase transitions}

The occurrence of phase transitions is probably one of the most remarkable phenomena that arises in asynchronous cellular automata: there exists a non-trivial value of the synchrony rate, the critical threshold, which separates two distinct qualitative behaviours of the system. We are now interested in measuring quantitatively this phenomenon.

\subsection{The Game of Life}

As pointed out in Sec. 3.1, the activity parameter reveals the existence of two distinct phases in the $\alpha$ - and $\beta$-synchronous Game of Life. For the $\alpha$-synchronism, the phase transition has been proved to be second-order [1], that is, if the macroscopic measures that describe the behaviour are continuous, their derivative curve is discontinuous for a critical value of the synchrony rate.

How to determine the critical synchrony rate $\alpha_{\mathrm{c}}$ or $\beta_{\mathrm{c}}$ ? A simple method to measure it consists in estimating the singularity point where the slope jumps from null to infinity, but for practical purposes, this method is rather imprecise as it introduces systematic biases. In order to reduce them, we follow a different protocol (see e.g. [5]):

1. We fix $\beta$, start from a random initial condition and let it evolve for a given number of steps.

2. We monitor the evolution of the order parameter for a long simulation time until we observe a sub-critical or super-critical behavior. In a loglog plot, a concave curve occurs for the passive phase (activity converges to zero) and a convex curve for the active phase (activity converges to a non-zero value). As we expect the order parameter to follow a power 

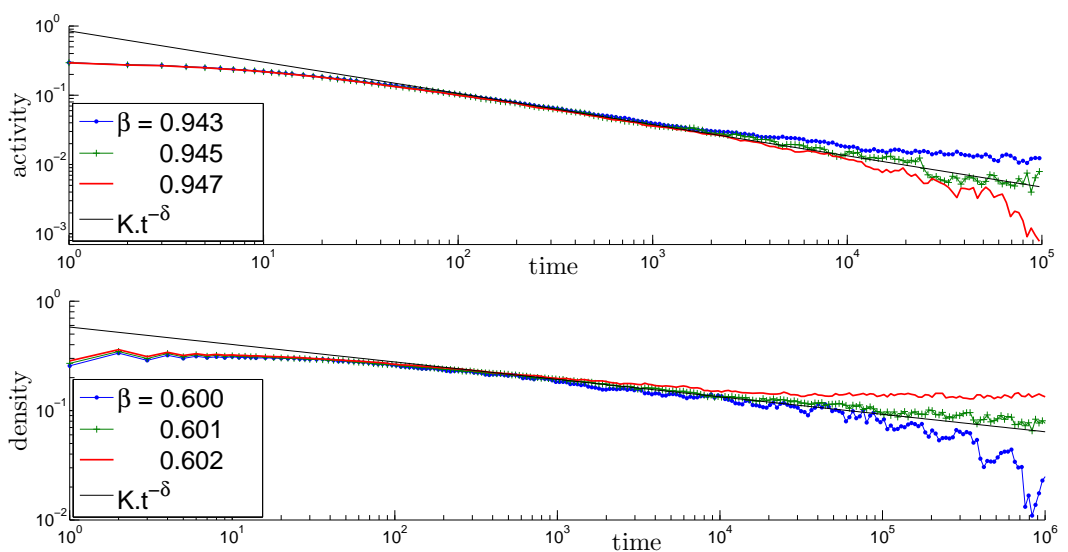

Figure 7: Phase transition analysis for the Game of Life (top) and ECA 50 (bottom). The straight lines follow a power law $f(t) \sim t^{-\delta}$. For the Game of Life, measures are averaged over 25 samples of size $800 \times 800$. For the ECA 50 , measures are averaged over 25 samples of size 20000. The straights line give the expected critical exponent for directed percolation: $\delta_{2 \mathrm{D}}=0.451$ (top) and $\delta_{1 \mathrm{D}}=0.1595$ (bottom).

law $K . t^{-\delta}$ near criticality, the curve at critical value should appear as a straight line of slope $-\delta$.

3. We repeat the experiment with a value closer to the critical point until a satisfactory precision is reached (here $10^{-3}$ ).

For the $\alpha$-synchronous Game of Life, it has been measured that $\alpha_{c}=0.9083$ and that the evolution of the order parameter of this value followed a power law in the form $K . t^{-\delta}$ with $\delta_{2 \mathrm{D}}=0.451$ [3], which is the expected critical exponent for the directed percolation universality-class in 2 dimensions [7]. Figure 7 shows that the measures for $\beta$-synchronous updating. They also confirm the directed percolation hypothesis, with $\beta_{c}=0.945$.

\subsection{Elementary Cellular Automata}

Among the 88 minimal ECAs, it has been observed that several ECA display second-order phase transition in their $\alpha$-synchronous version. These rules were proved to belong to the directed percolation class [2], and have been divided into 3 distinct subclasses:

- rules $18,26,50,58,106$ and 146 are the $\mathrm{DP}_{\text {hi }}$ class, for which the active phase of density (respectively the passive phase) occurs for $\alpha>\alpha_{\mathrm{c}}$ (resp. $\left.\alpha<\alpha_{\mathrm{c}}\right)$.

- rules 6,38 and 134 are the $\mathrm{DP}_{\text {low }}$ class, where active and passive phases are inverted. 
- rule 178 is the sole element of the $\mathrm{DP}_{2}$ class, where the density is stable in average but for which a phase transition appears for the 01-block-density.

Our observations show that these subclasses react differently to $\beta$-synchronism:

1. The rules of the $\mathrm{DP}_{\mathrm{hi}}$ class have shown little behavioural change between the two types of asynchronism: the phase transition appearing for the density parameter in Fig. 5 is conserved.

For $\alpha$-synchronism, the same protocol was applied to ECA 50 [2], and displayed good evidence of a directed percolation phenomenon for $\alpha_{\mathrm{c}}=$ 0.6282 .

For $\beta$-synchronism (see Fig. 7), it appears the behaviour at critical synchrony rate $\beta_{\mathrm{c}}=0.601$ is in good agreement with a power law of critical exponent $\delta_{1 \mathrm{D}}=0.1595$, the expected value for the directed percolation.

2. Surprisingly enough, no phase transition was observed for the three rules of the $\mathrm{DP}_{\text {low }}$ class in $\beta$-synchronism (see ECA 6 in Fig. 5), leaving a constant null-density convergent phase for any value of $\beta$.

3. Finally, the ECA $178\left(\mathrm{DP}_{2}\right.$ class) reproduced a similar plot profile for the 01-block-density (see ECA 178 in Fig. 5).

These first results show how rich the study of $\beta$-synchronism can be, and that although $\alpha$ - and $\beta$-synchronism are intuitively similar in their mechanisms, their effects may differ radically.

\section{Discussion}

This paper presented a formalism for a new type of asynchronous updating in cellular automata, the $\beta$-synchronism, based on the disruption of information transmission between cells. We compared this perturbation to $\alpha$-synchronism and observed from a macroscopic point of view that $\alpha$ - and $\beta$-synchronism had similar effects. It was observed that $\beta$-synchronous updating also produces phase transitions, but for a smaller set of rules than $\alpha$-synchronism. In particular, we remarked that there was no phase transition for the three ECA of the $\mathrm{DP}_{\text {low }}$ class but we have no explanation for this phenomenon so far.

By studying ECA 50 more closely, we could exhibit an example for which a macroscopic parameter behaviour was similar for both types of asynchronism, but for which novel lower-scale properties were observed.

This extension is a first step towards a unified view of asynchronism for cellular automata, based on the idea of cell cycles. There exist plenty of other ways the cellular activity can be perturbed. This raises the question of how the space of perturbations can be described.

Although this paper focused on cellular automata, the idea behind the $\beta$ synchronism can be transposed to other synchronous collective systems, such as lattice-gas cellular automata, neural networks or multi-agent systems. By 
extending the range of variations applied to the definition of the discrete dynamical systems, can we perfect our understanding of the robustness of natural systems? Can we gain some insight from their mechanisms to improve the design of spatially-extended computing models?

\section{References}

[1] Hendrik J. Blok and Birger Bergersen, Synchronous versus asynchronous updating in the "Game of Life", Physical Review E 59 (1999), 3876-9.

[2] Nazim Fatès, Asynchronism induces second order phase transitions in elementary cellular automata, Journal of Cellular Automata 4 (2009), no. 1, $21-38$

[3] Nazim Fatès, Does Life resist asynchrony?, Game of Life Cellular Automata (Andrew Adamatzky, ed.), Springer, 2010, pp. 257-274.

[4] Nazim Fatès and Michel Morvan, An experimental study of robustness to asynchronism for elementary cellular automata, Complex Systems $\mathbf{1 6}$ (2005), 1-27.

[5] Peter Grassberger, Synchronization of coupled systems with spatiotemporal chaos, Physical Review E 59 (1999), no. 3, R2520.

[6] Carlos Grilo and Luís Correia, Effects of asynchronism on evolutionary games, Journal of Theoretical Biology 269 (2011), no. 1, 109 - 122.

[7] Haye Hinrichsen, Nonequilibrium critical phenomena and phase transitions into absorbing states, Advances in Physics 49 (2000), 815-958.

[8] Ferdinand Peper, Teijiro Isokawa, Yousuke Takada, and Nobuyuki Matsui, Self-timed cellular automata and their computational ability, Future Generation Computer Systems 18 (2002), no. 7, 893 - 904. 